\title{
Traffic Congestion Model in India by Shock Wave Theory
}

\author{
Tsutomu TSUBOI ${ }^{\mathrm{a}, 1}$ \\ ${ }^{a}$ New Business Creative Division, Nagoya Electric Works Co. Ltd., Japan
}

\begin{abstract}
This manuscript is a series of traffic flow analysis in India and it describes traffic congestion model by using shockwave theory from fluid mechanism. This is unique study for emerging country India traffic flow analysis during more than one month in October 2020. In order to chaotic traffic flow analysis in India, author observes one moth traffic flow data from the traffic monitoring cameras in 26 locations in a city where it is one of major city Ahmedabad in Gujarat states of India. In terms of traffic congestion, it is sued occupancy parameter of traffic flow data which indicates road occupancy percentage by vehicles. By using shock wave theory, author defines two traffic congestion model which are "forwarding traffic congestion" model and "stacking traffic congestion" model. These models explain traffic congestion condition and it is able to provide hint for solving traffic congestion problem in India.
\end{abstract}

Keywords. Traffic congestion, shock wave, traffic flow

\section{Introduction}

The traffic congestion becomes issues especially in emerging countries such as India these days, which causes $\mathrm{CO} 2$ emission under growing number of vehicles because of economic growth. This issue is not only in India but also there is potential in any emerging countries. However it is big challenge to understand its traffic congestion because there is not enough traffic data and analyze traffic congestion mechanism. It is only clear condition, just too much vehicles and not enough infrastructure improvement such as road construction, traffic management system installation.

In general, traffic flow analysis has been started in 1933 when Greenshields B.D. [1] introduced traffic flow theory from his observation at the 13th Annual Meeting of the Highway Research Board. This theory is widely used for any traffic flow analysis and it also established as traffic flow fundamental equation from fluid flow analysis [2]. Therefore fluid flow theory is valid for considering traffic flow at the fundamental analysis. Therefore, we take shock wave theory for Indian traffic congestion analysis in this study.

After Greenshields traffic flow theory has been introduced, many traffic flow analysis has used and its theoretical validation has been confirmed. This is useful for understanding traffic flow characteristics such as traffic density $(k)$ to average vehicle speed $(v)$ characteristics, traffic density $(k)$ to traffic volume $(q)$ character etc. From these

${ }^{1}$ Corresponding Author, Tsutomu Tsuboi, New Business Creative division, Nagoya Electric Works Co. Ltd., 29-1 Ama-shi, Aichi, 490-1294, Japan; E-mail: t_tsuboi@nagoya-denki.co.jp. 
traffic flow analysis is valid to understand traffic flow condition but it is difficult to know micro traffic characteristics such as traffic congestion. Therefore, in traffic flow analysis, there are several challenges to use other flow mechanism about traffic congestion. One of flow mechanism is shock wave theory from fluid flow theory. This shock wave theory is also useful for explaining about traffic congestion in orderly traffic flow such as at highway traffic because moving behavior of vehicles is almost liner relationship between traffic speed and traffic volume. Therefore, it is challenge for make analysis at urban traffic flow, especially under developing countries such as India. The traffic condition in India as we know, is very chaotic and un-orderly driving behavior.

In the next section 2, it describes traffic flow observation location and environment. And the shock wave theory and occupancy parameter in the section 3 . In the section 4 , real traffic congestion analysis result is explained. In the section 5, this study summary and conclusion are described.

\section{Observation environment,}

This study is joint research project between Japan and India as government funded for five years since April 2017. This project focuses on Low Carbon Society development for transportation in major cities of India.

\subsection{Field}

The field is Ahmedabad city in Gujarat states of India and it locates in the west part of India. Its population is about 6 million at 2011. The current population is over 8 million [3] in 2018 from 5 million in 2011 and the number of vehicles is about 4 million in 2017 [4]. More than $70 \%$ vehicle is two wheelers, which is typical percentage in developing countries.

The official city profile of Ahmedabad is shown in Table 1.

Table 1. The city profile of Ahmedabad from local government "Ahmedabad Municipal Corporation or AMC.

\begin{tabular}{ll}
\hline Co-ordinates: & $23.03^{\circ} \mathrm{N} 72.58^{\circ} \mathrm{E}$ \\
\hline Area: & $466 \mathrm{Sq} \cdot \mathrm{km}$. (year 2006) \\
Population: & $55,77,940$ (year 2011 Census) \\
Density: & $11,948 / \mathrm{sq} \cdot \mathrm{km}$ \\
Literacy Rate: & $89.60 \%$ \\
Average Annual Rainfall: & $782 \mathrm{~mm}$ \\
Popularly known as: & Amdavad \\
STD Code: & 079 \\
\hline
\end{tabular}




\subsection{Observation Environment}

In this study, we have 31 traffic monitoring cameras in the city and its traffic data is collected at every minutes 24 hours per day. The camera's installation points are shown in Figure 1.

The camera's installation points are shown in Figure 1. The number shows each traffic monitoring camera location. Two digit number cameras are located in west side of block along with "132 Feet Ring Road" where there are many office, shops and market. The four digit number cameras are located near the river "Sabarmti River" so called River Front and new development area.

The River Front is control area by city government and it is not allowed to enter by auto-rickshaw and city bus. This road is currently used for a way to airport from city center. The main study location is on these both area because it is highly developing for business and the traffic grows heavily year by year. And there is another aspect which is metro development plan. The blue and red line in the map are Metro line which are under development by 2023 .

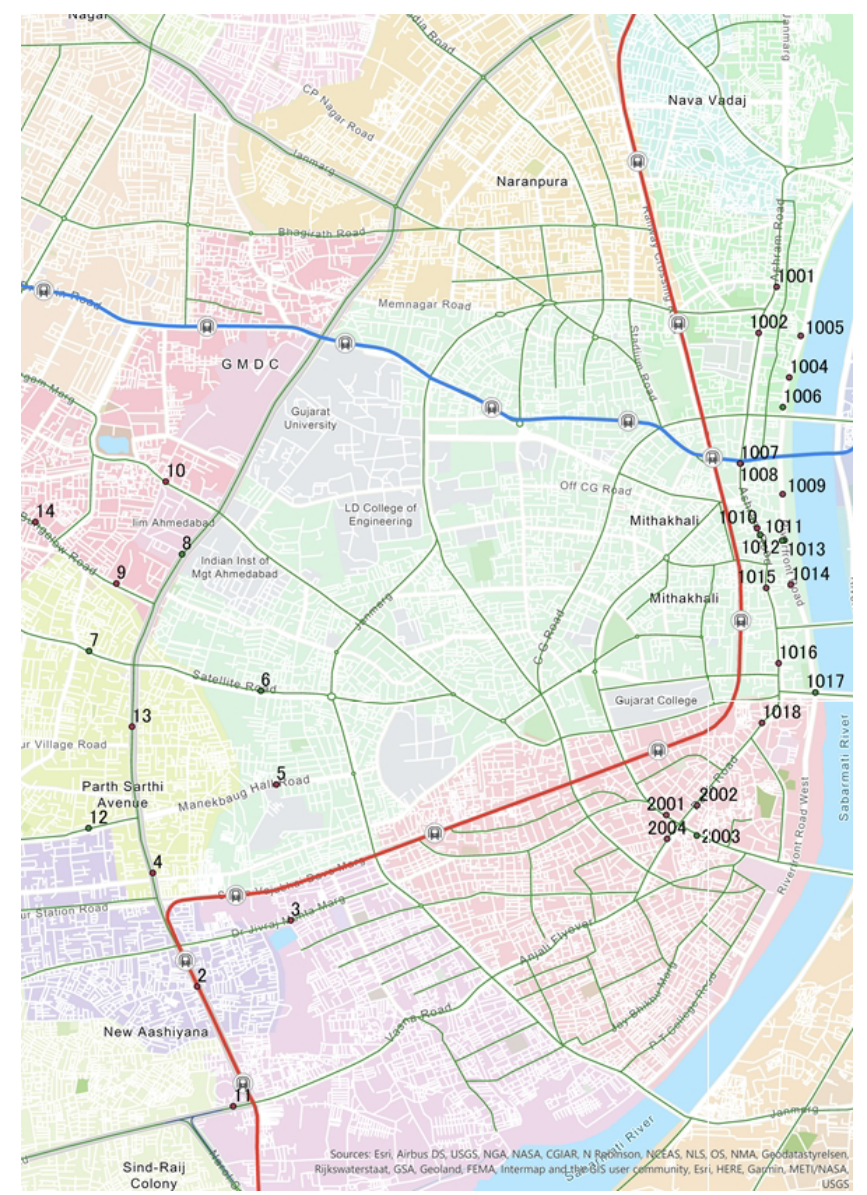

Figure 1. Observation environment and location of traffic monitoring cameras. 
In traffic flow analysis, there are two types of research, one is micro traffic flow analysis for example some major intersection traffic flow such as the area of camera \#2001 through camera \#2004-Paldi junction [5]. The other one is to focus on over all traffic condition analysis such as overall city traffic condition analysis [6]. In this study, we focus on traffic congestion not only micro area but also macro view. Especially macro view traffic congestion is important to know because traffic congestion is caused by several geographical environmental reason.

\section{Traffic Flow Theory}

In this section, we explain two traffic theory. One is shock wave and the other ones is occupancy parameter as traffic congestion condition parameter.

\subsection{Shock Wave}

The shock wave theory comes from fluid mechanism originally but it becomes common for traffic flow analysis [7].

When there are two phases condition of a road which is illustrated in Figure 2, the number of vehicle passing boundary $\mathrm{S}$ is same between cross section A1 and cross section A2. When its number of vehicle at boundary $\mathrm{S}$ is $N$, Equation (1) is established. The road model in Figure 2 represents typical traffic congestion situation where driving vehicles go from wide area A1 to narrow space A2. The narrow point A2 is no necessary physically narrow because one traffic congestion occurs, front vehicles do not go smoothly, which is equivalent with physically narrow road.

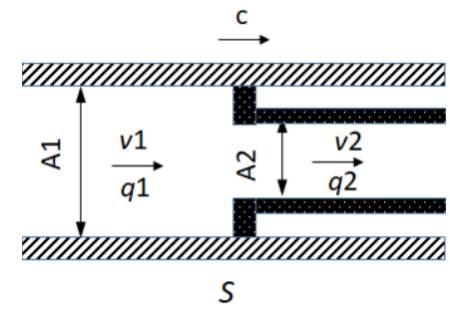

Figure 2. Traffic Flow Shock Wave model.

$$
N=\left(v_{1}-c\right) k_{1} t=\left(v_{2}-c\right) k_{2} t
$$

where $(c)$ is moving speed at boundary $\mathrm{S},(k)$ is traffic density at each area 1 and 2 , $(t)$ is time, and $(v)$ is vehicle speed at each area.

From Equation (1), Equation (2) is established.

$$
\left(v_{1}-c\right) k_{1}=\left(v_{2}-c\right) k_{2}
$$

Therefore, Equation (3) is established. 


$$
c=\frac{v_{2} k_{2}-v_{1} k_{1}}{k_{2}-k_{1}}=\frac{q_{2}-q_{1}}{k_{2}-k_{1}}
$$

When $q_{2}-q_{1}=\Delta q$ and $k_{2}-k_{1}=\Delta k$, Equation (3) is shown as Equation (4) by small changes of each parameters.

$$
\mathrm{c}=\frac{\Delta q}{\Delta k}=\frac{d q}{d k}
$$

When it takes the differentiation by $(k)$ of both side, Equation (5) is established because of $q=k v$.

$$
\mathrm{c}=\frac{d q}{d k}=v+k \frac{d v}{d k}
$$

From Greenshields equation, $\mathrm{d} v / \mathrm{d} k<0$ is established. Then Equation (6) is established.

$$
\mathrm{c}<v
$$

Therefore shockwave is transferred from the preceding vehicle in traffic flow to the subsequent vehicle. From traffic flow theory, critical traffic density is $k_{\mathrm{c}}$ and jam traffic density is $k_{\mathrm{j}}$, Equation (7) is established and shock-wave is transferred forward direction and backward direction.

$$
\left.\begin{array}{ll}
\mathrm{c}>0 \\
\mathrm{c}=0 \\
\mathrm{c}<0
\end{array} \quad \begin{array}{l}
\text { where } 0 \leq k<k_{c} \\
\text { where } k_{c} \leq \mathrm{k}<k_{j}
\end{array}\right\}
$$

From Equation (7), positive value (c $>0$ ) of shock wave means vehicle in front of section $\mathrm{S}$ becomes bottleneck by its congestion because the vehicle before section $\mathrm{S}$ has relative space between vehicles. On the other hand, negative value $(c<0)$ of shock wave means vehicles before section $\mathrm{S}$ becomes bottleneck by congestion because the vehicle after section $\mathrm{S}$ has relative space between vehicles. Figure 3 shows this condition as illustration of traffic congestion model using the model of Figure 2.

When shock wave value equals to 0 , there are two meanings - traffic has no congestion and moves smooth or traffic has completely congested and no movement.
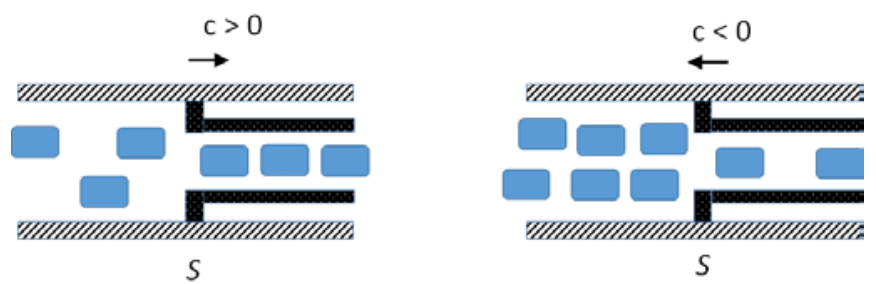

Figure 3. Traffic Congestion model

Therefore, $\mathrm{c}>0$ condition is defined "forward moving traffic congestion" and $\mathrm{c}<0$ condition is "stacking traffic congestion". From our experience, when traffic congestion starts from "forward moving congestion" to "stacking traffic congestion". 


\subsection{Occupancy}

In terms of traffic congestion parameter from traffic flow, occupancy parameter is used in general. The occupancy (OC) is defined the percentage ratio between total measurement time $(t)$ of the vehicles to a certain block of road section under certain moment. The formula is shown in Equation (6)

$$
O C=\frac{1}{T} \sum_{i} t_{i} \times 100(\%)
$$

where $T$ is time of measurement, $t_{\mathrm{i}}$ is detected time of vehicle $i$ [8].

When number of existing vehicle a certain section is $\mathrm{N}$, average length of vehicle is $\bar{l}$, Equation (7) is given.

$$
O C=100 \frac{q}{v} \bar{l}=100 \mathrm{k} \bar{l}
$$

Therefore occupancy (OC) is proportional to traffic density $(k)$ and traffic volume $(q)$. From our previous study, occupancy shows actual traffic congestion condition [9]. And traffic congestion condition is normally $25 \%$ value.

\subsection{Usage of traffic theory}

From the previous traffic flow theory - shock wave and occupancy, the occupancy parameter indicates traffic congestion condition in general. Therefore, occupancy is the first choice parameter for judging traffic congestion condition from its traffic data. After identifying traffic congestion point in time basis and location basis, the next step focuses on traffic congestion patterns by using shock wave mechanism. In the previous section 2.1 , there are two types of shock wave condition and they explain about traffic congestion model - forward forming model and backward recovery model. The forward forming shock wave shows the first stage of traffic congestion condition when its traffic congestion starts at the road. And the backward recovery shock wave shows heavy traffic congestion condition and vehicles which approach to the congestion area becomes stack situation because the front vehicles are not able to move because of its traffic congestion.

This traffic congestion analysis process is main research contexts in this study.

\section{Discussion}

Based on traffic measurement of the traffic monitoring cameras in Figure 1, Table 2 shows all occupancy value which is equal and greater than $25 \%$ condition. From October 2020 measurement data, the congestion occurs from 18:00 to 20:00 time frame.

Table 2. The occupancy measurement data in October 2020 at each location

\begin{tabular}{rrrrrrrr}
\hline Time & \multicolumn{1}{r}{} & \multicolumn{1}{c}{18} & 19 & 20 & 21 & 22 \\
\hline Camera & 2 & $\mathbf{3 3}$ & $\mathbf{5 9}$ & $\mathbf{4 7}$ & $\mathbf{3 7}$ & $\underline{\mathbf{2 6}}$ \\
\cline { 2 - 8 } & 3 & 12 & 18 & 15 & 12 & 9 \\
\cline { 2 - 8 } & 4 & 19 & $\mathbf{2 9}$ & 24 & 20 & 15 \\
\cline { 2 - 8 } & 5 & 16 & 18 & 14 & 11 & 9 \\
\cline { 2 - 8 } & 6 & 3 & 4 & 4 & 5 & 5 \\
\hline
\end{tabular}




\begin{tabular}{rrrrrr}
7 & 15 & 20 & 15 & 12 & 10 \\
\hline 8 & 13 & 14 & 9 & 10 & 6 \\
\hline 9 & 20 & 25 & 21 & 18 & 17 \\
\hline 10 & 11 & 13 & 9 & 8 & 6 \\
\hline 11 & 24 & 35 & 25 & 19 & 15 \\
\hline 13 & 15 & 21 & 16 & 12 & 9 \\
\hline 1001 & 14 & 18 & 14 & 12 & 10 \\
\hline 1002 & 12 & 15 & 11 & 9 & 8 \\
\hline 1003 & 18 & 21 & 16 & 12 & 9 \\
\hline 1004 & 6 & 8 & 6 & 6 & 5 \\
\hline 1006 & 11 & 16 & 10 & 7 & 5 \\
\hline 1007 & 9 & 10 & 7 & 5 & 5 \\
\hline 1008 & 13 & 15 & 12 & 9 & 7 \\
\hline 1009 & 8 & 11 & 8 & 7 & 5 \\
\hline 1010 & 13 & 16 & 12 & 10 & 10 \\
\hline 1011 & 11 & 13 & 10 & 8 & 8 \\
\hline 1013 & 9 & 10 & 7 & 6 & 5 \\
\hline 1014 & 7 & 9 & 6 & 5 & 5 \\
\hline 1018 & 19 & 27 & 22 & 19 & 17 \\
\hline 2003 & 14 & 22 & 19 & 16 & 14 \\
\hline 2004 & 16 & 23 & 19 & 16 & 15
\end{tabular}

In Table 2 , the highlighted occupancy value is equal and greater than $25 \%$, which means its traffic condition is congested. There are five location in Figure 1.In Table 2, underline value is negative value of shockwave which is "stacking traffic condition". In most case of traffic congestion occurs at 19:00 time frame. Based on this results, Figure 4 shows overall traffic density geographical image by ArcGIS tools. The value of Traffic density is vehicle $/ \mathrm{km} \cdot$ lane. We see the location camera \#11, \#2, \#4 is most traffic congested area at 19:00 and most of location is "forward traffic congestion" condition.

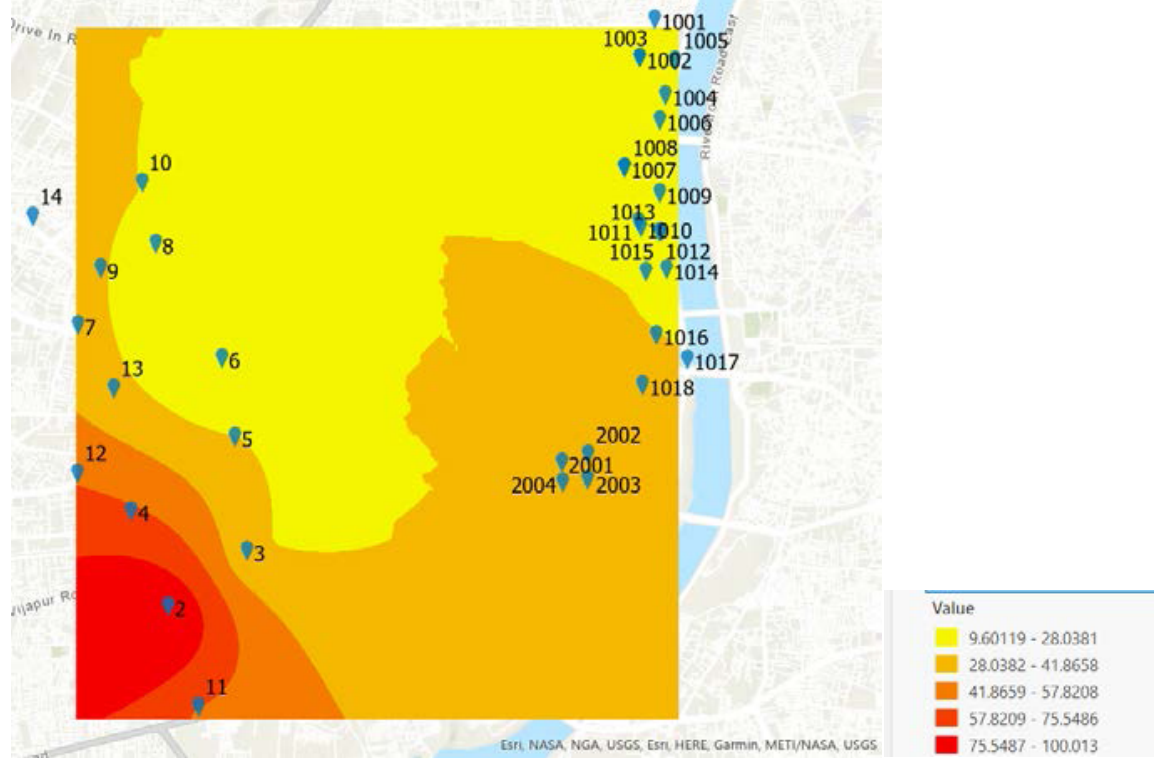

Figure 4. Traffic Density (vehicles/km·lane) in Ahmedabad at 19:00 


\section{Summary and Conclusion}

In this study, we use shock wave analysis for Indian traffic congestion condition and it enable to identify the traffic congested time frame. In October 2020 traffic, the most traffic congested time is from 18:00 to 22:00 and particularly 19:00 is most crowed time. And the traffic congestion model is "forwarding traffic congestion" which means traffic congestion starts. On the other hand, "stacking traffic congestion" condition is very dedicated which happened at Camera \#11 at 19:00 and Camera \#2 at 22:00.

From this study, it is able to define clear traffic congestion time frame and location. It is necessary to study the reason why 18:00 to 22:00 is traffic congested time zone and the congested location is almost same area in Ahmedabad where is Camera \#2 location. The area Camera \#2 is commercial zone and there is big market in this area. The detail investigation of traffic congestion is future work.

As for conclusion, we show traffic congestion analysis in India by using traffic theory two parameters which is occupancy parameter for the indication about traffic congestion condition and shock wave parameter for the modeling traffic congestion condition. Those two parameter are useful to define traffic congestion condition for analyzing chaotic Indian traffic congestion. This method brings Indian traffic congestion mechanism how its traffic congestion occurs. It is necessary to analyze more area of Indian traffic data such as other month time period of traffic data in the next study. And it is also necessary to check the actual traffic congestion condition to visit on-site observation especially most congested point and time frame which we have already had some result of this study.

\section{Acknowledgement}

This research is part of SATREPS program 2017 (ID: JPMJSA160 between India and Japan

\section{References}

[1] Kuhne R.D, Foundations of Traffic Flow Theory I: Greenshields' Legacy - Highway Traffic. Available from https://www.krbalek.cz/For_students/mds/clanky/Greenshields.pdf, [Accessed: 2021 Sep.]

[2] Greenshields B.D., A Study of Traffic Capaci, Proc.H.R.B, 1935 , 14, pp.448-477.

[3] Population of India [Internet] 2020 Available From: https://indiapopulation2019.com/population-ofahmedabad-2019.html [Accessed: 2020-08-21]

[4] Registered number of vehicles Ahmedabad India FY 2006-2017 Available From: https://www.statista.com/statistics/665754/total-number-of-vehicles-in-ahmedabad-india. [Accessed: 2020-08-21]

[5] Tsuboi T., Traffic Congestion "Gap" Analysis in India, 7th International Conference on Vehicle Technology and Intelligent Transport Systems, 2021

[6] Tsuboi T, Visualization and Analysis of Traffic Flow and Congestion in India, Infrastructures (ISSN $2412-$ 3811) on 01 March 2021

[7] Gupta A., Katiyar V.K., Analyses of shock waves and jams in traffic flow, Journal of Physics A Mathematical and General 38(19):4069, April 2005.

[8] Kawakami.S, Matsui.H, Transport Engineering, Morikita Publishing Co., 2007, pp.102-103

[9] Tsuboi T., Traffic Congestion Visualization by Traffic Parameters in India, The 2nd International Conference on Intelligent Control and Computing (ICICC) 2019. 